\title{
Die Namen der Monate bei den Surgut-Ostjaken
}

Aus den verschiedenen Surgut-ostjakischen Mundarten wurden nur aus der Tremjugan-Mundart die Namen der Monate, und zwar 13, teils unübersetzt, veröffentlicht, vgl. KT. 998b999a. In Paasonens grammatikalischen Aufzeichnungen aus der Konda-Gegend befinden sich anf der vorletzten Seite in zwölf Zeilen zwölf Syntagmen, die die Namen der Monate im surgutschen Kreise darstellen (Kuukausien nimet Surgutin tienoiden ostjakeilla). Eine russische Übersetzung ist nur in der ersten Zeile, eine finnische Erklärung in der siebenten zu finden. Zehn der Namen stimmen ganz und gar mit den von Karjalainen aufgezeichneten überein, zwei sind im grossen und ganzen ähnlichgeartet und der dreizehnte ron Karjalainen aufgezeichnete Name fehlt. Die Reihenfolge der Monatsnamen ist in den Aufzeichnungen der beiden Forscher bis anf zwei Abweichungen dieselbe, die Bedentung der ostjakischen Namen ist jedoch bei den Gewährsmännern aus den verschirdenen Gegenden des surgutschen Kreises nicht dieselbe.

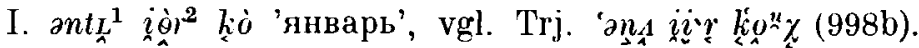
Dieser Monatsname ist in Paasonens Aufzeichnung dem Namen des letzten Monats ähnlich, s.u. XII. Im Wörterbuch von Karjalainen-Toivonen folgt nach diesem Monatsnamen 'aij $\underset{i}{i} \underset{x}{\text { ? }}$ ?

1 Zum Verhältnis der hier benutzten Transkription von Paasonens Aufzeichnungen, die mit jener seines Wörterbuches identisch ist, und der des Manuskriptes vgl. PD. XIII-XV.

${ }^{2}$ Der Buchstabe $\dot{\theta}$ wurde in die Transkriptionstabelle von Dows ER (PD. XIV) nicht aufgenommen, dort ist nur $\dot{\theta}$ zu finden, welches im Wörterbuch durch $\dot{\theta}$ ersetzt worden ist. Höchstwahrscheinlich ist auch $\dot{\theta}$ durch $\dot{\theta}$ zu ersetzen. Da aber bei der l'bersetzung des ersten und des Jetzten Monatsnamens gewisse Hypothesen nötig sind, behalte ich bei diesem einen Worte die Originaltranskription bei. 
k: $0^{u} \%$, mit der Anmerkung: "Toisessa kohden on nämä kaksi ensiksimainittua kuukauden nimeä mainittu päinvastaisessa

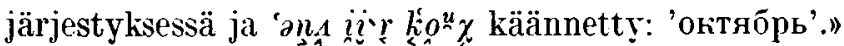

Die Bedeutung des ersten Wortes ist 'gross' (PD. 141, KT. 61b). Die Wörter J jàr 'Tieropfer' (340) und Trj. ii 're 'Opfer' (182a) entsprechen einander sicherlich auch in den beiden Monatsnamen. Das letzte Wort ist in Paasonens Aufzeichnungen J kiò 'Mann, Gatte' (548), bei Karjalainen Trj. Ki $0^{u} \chi$ 'lang' (285̃b). Da die folgenden zehn MIonatsnamen gänzlich identisch sind. und die ersten zwei Wörter auch in der Benennung des ersten und des letzten Monats einander entsprechen, kann angenommen werden, dass den Wörtern $\mathrm{J}$ kì, Trj. $k^{\prime} \mathfrak{o}^{\circ} \%$ auch e in Wort zu Grunde lag, welches in den verschiedenen Gegenden des surgutschen Kreises rolksetymologisch auf verschiedene Weisen verändert worden ist, vgl. noch XII. Man würde hier ein Wort mit der Bedeutung 'Monat' erwarten. Paasonen hat nur an der Konda ein solches Wort aufgezeichnet, welches dem Sinne ebenso wie seinem Lautbestand nach gänzlich hierher passen könnte: ұaw 'kuukausi : Monat' (PD. 453). Der Annahme, dass dieses Wort einst auf grösserem Sprachgebiet unter den Ostjaken bekannt war, steht nichts im Wege. Es gehört ja zu dem finnisch-ugrischen, sogar uralischen Bestandteil des ostjakischen Wortschatzes, rgl. ung. hó, har'Monat', mord. E, M kov, E kon 'Mond; Monat', fi. kuu 'Mond'. kuu(kausi) 'Monat', ? IpPi. güjiidit 'nousta (kuu)', samK kìi, kli, ki 'Mond; Monat, Koib. küii, kyii (PaAsonen Xy K 39 S. 347, Beitr. S. 28; Beke Nyr. 38 S. 451; Szinnyei $\mathrm{NyH}^{4-7}$, FgrSprw. ${ }^{1-2}$; SетёLё JSFOu 30:5 S. 41; Fonos Nỵr. 65 \$. 158; Bárczi Szúfsz.; E. IthoNen FLF 30 \$. 50: Collixder FUY; Tolvonex-ItkoNex-Joki SKES). Das syrjänische Lehnwort DN țį̧šs 'Mond; Monat' (Torvosex FUF 32 s. 91, mit Angabe der früheren Literatur), welches sich ausser in den Nord- und Südmundarten auch im Surgutgebiet verbreitet hat (Trj. $t^{\prime}(\bar{l}, 12 s$ KT. 998b, J tèlas PD. 2544), konnte das urtümliche uralische Wort - welches Paasonen nur in der Folklore angetroffen hat ( $\mathrm{Y} y \mathrm{~K} 39 \mathrm{~s} .348$ ) - rerdrängen. Das veraltete und unverständlich gewordene Wort $\chi a c$ oder in Patkanows Aufzeichnungen $\chi o u$ - konnte volksetymologisch mit 
dem Worte 'lang' zusammengestellt werden. Sicherlich stammen die von Fuchs-Fokos verbesserten falschen Übersetzungen (Nyr. 65 S. 158) bei Patkaxow nicht ron ihm, sondern von seinen Gewährsmännern: wenn in den in Frage stehenden Trj. Jonatsnamen $k_{2} 0^{*} \%$ 'lang' aus einem Wort mit der Bedeutung 'Monat' herzuleiten ist, so zeugt diese rolksetymologische Deutung auch hierfür. Wahrscheinlich kömnen gewisse Züge des primitiven Volksglaubens. die wohlbekannten Personifikationen im Glauben der Heiden, eine genügende Erklärung dafür liefern, warum das erschlossene Wort 'Monat' am Jugan nicht mit kouc 'lang', sondern mit lic 'Mann' ersetzt worden ist.

Der folgende Monatsname ist bei Karjalainen Trj. țợ.joy

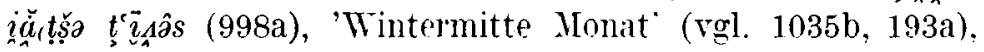
dieser Name ist bei Paasonen nicht belegt.

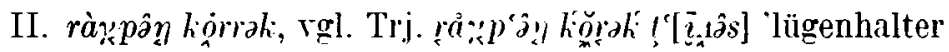
Adler (Monat)' (PD. 1968, 846, KT. 798b. 42+a).

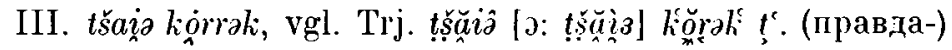
'wahrer Adler (Monat)' (PD. 846, K'T. 925a, 4ㄴa).

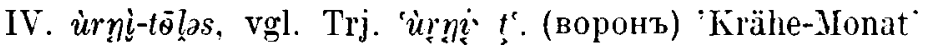
(PD. 2904, wo aber vùnไ zu finden ist 2544. KT. 243a).

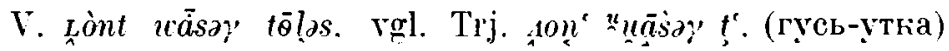
'Gans Ente-Monat' (PD. 2671, 2962, KT. 1077a. 249a).

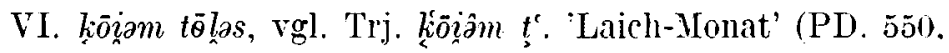
wo der betreffende Name aus der K. und aus der .J. Mundart mit der Übersetzung 'cin Frühlingsmonat. Y[ai (russ.)' ang+führt ist, KT. 285a).

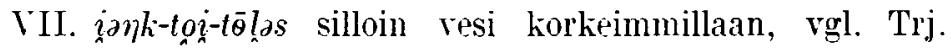

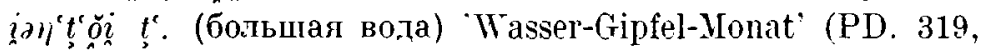
2508, KT. 161a, 966b).

VIII. $u \dot{a r}-t \bar{\theta} l \partial s$, vgl. Trj. "uarr $t^{c}$. (зао̆оръ) '(Sommer)dammMonat' (PD. 2956), 'Wehr-Monat' (KT. 233a).

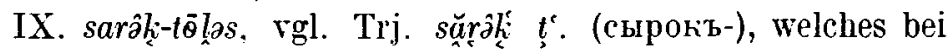
Karjalainen erst nach dem hier unter $\mathrm{X}$. angeführten folgt, 'Coregonus vimba-Monat' (PD. 2085), 'Zährte-Monat' (KT. 871a).

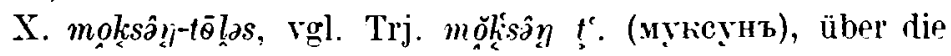
Reihenfolge bei Karjalainen s. u. IX. 'Coregonus moksunIonat' (PD. 1243, KT. 515a). 
XI. soy-tōlas, vgl. Trj. say ț. (mateen-kutukuu) 'Quappenmonat', nach der finnischen Erklärung 'Quappen-Laich-Monat' (PD. 2157, KT. 835a).

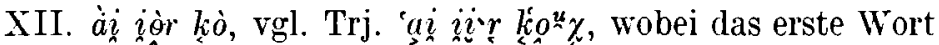
die Bedeutung 'klein' hat (PD. 57, KT. 3b), und bezüglich der weiteren Glieder auf $\mathrm{I}$. hingewiesen werden muss.

Auf die Ungewissheit der Bedeutungen der Monatsnamen weist nicht nur der Unterschied in der Reihenfolge der Aufzeichnungen von Karjalainen und Paasonen, sondern wie aus der Darstellung hervorgeht, ist der unter VI. angegebene Monat bei Paasonen in diesem Zusammenhang .Juni, nach dem Wörterbuch Mai.

Edith VÉRTES 